\title{
Chapter 9: Implementing and Measuring the Efficacy of Biosecurity and Dual-use Education
}

\author{
JAMES REVILL AND GIULIO MANCINI
}

Teaching security-related issues to science students is increasingly salient in the international security discourse, yet despite the calls for greater education, research conducted by the authors and others contributing to this volume (as in the chapters by Friedman and Minehata and Shinomiya) demonstrates that the calls for, and interest in, biosecurity education have too infrequently been converted to commensurate activity at the level of the practising life scientist. Indeed, it remains clear from previous chapters that there are currently limits to the extent of biosecurity education and the process of promulgation and implementation of education has been slow, something stymied by practical, ethical and philosophical considerations which need to be addressed in the process of moving from aspirational declarations by states and other organisations to concrete action (see the chapter by Johnson).

The purpose of this chapter is to posit some partial responses to the questions posed in the introduction, specifically questions related to the 'Who, What and How' of education. These will be based on the authors' experiences derived from a joint project between the Landau Network Centro Volta (LNCV) and Bradford Disarmament Research Centre (BDRC) on sustainable biosecurity and dual-use education. This project represents one experimental applied approach to the implementation and promulgation of a specific element of biosecurity education that focuses on the 'wider ethical/arms-control issues' mentioned earlier by Rappert. In this regard, biosecurity in the context of this chapter relates less to the notion of laboratory biosecurity. Rather, is much more closely linked to concerns over the limited awareness amongst scientist both of the existence of measures intended to prevent and prohibit the malign exploitation of the life sciences, and how benignly intended research could be misapplied.

This chapter firstly provides a brief elaboration of the phases of activity in the LNCV-BDRC project, beginning with a gap analysis of university curricula in Europe and proceeding with the construction of a collaborative 
Biosecurity Education Network. Secondly, the chapter outlines the process of implementation testing using tailored material that was presented primarily to life-science students under the supervision of network members working within a number of departments in European universities. Thirdly, this chapter provides information on some of the lessons learned over the course of implementation tests, before underlining the vital importance of other complementary intervention points which could be addressed in the process of moving from aspirational statement of interest to concrete activity.

\section{Biosecurity Education Survey}

The joint LNCV-BDRC project was implemented with the intent of firstly developing an understanding of the extent of, and attitudes to, education on wider ethical/arms-control and dual-use issues within life-science degree courses. Also underlying this objective was an aim to identify means and methods through which biosecurity education could be more effectively promulgated across the life-science community in Europe. In this sense, the LNCV-BDRC project was initiated with a focus on university students. Whilst recognising that other targets could be considered for inclusion, engaging students was deemed particularly important because the development of advanced biological weapons is likely to require some degree of sophisticated scientific capability. This logically points advocates of education towards a primary target group consisting of individuals trained in the life sciences to at least undergraduate level. Moreover, engagement with this sector could be particularly beneficial in the long term in feeding into a sustainable culture of responsibility built by the next generation of life scientists.

In the first phase of the LNCV-BDRC project, the aim was to produce a gap analysis which assessed the extent of, and attitudes to, biosecurity-related content in universities across Europe using a sample of 142 degree courses (undergraduate and master's level) at 57 universities in 29 countries. ${ }^{1}$ This phase involved an assessment of the existence and extent of biosecurity-related content through the development of sample and search terms and the implementation of an investigation into available content (including online programmes of study and syllabi); but also the identification of key individuals within departments with whom we subsequently confirmed the provisional results and elicited an understanding of attitudes through follow-up correspondence.

\footnotetext{
1 The Member States of the European Union but also Norway and Switzerland. The sample was selected on the basis of a balance between international ranking and geographical representation, see Mancini, G. and Revill, J. 2008, 'Fostering the Biosecurity Norm: Biosecurity Education for the Next Generation of Life Scientists', Working paper produced for the Landau Network-Centro Volta, available:http://www.centrovolta.it/landau/2008/11/20/ FosteringTheBiosecurityNormBiosecurityEducationForTheNextGenerationOfLifeScientists.aspx.
} 


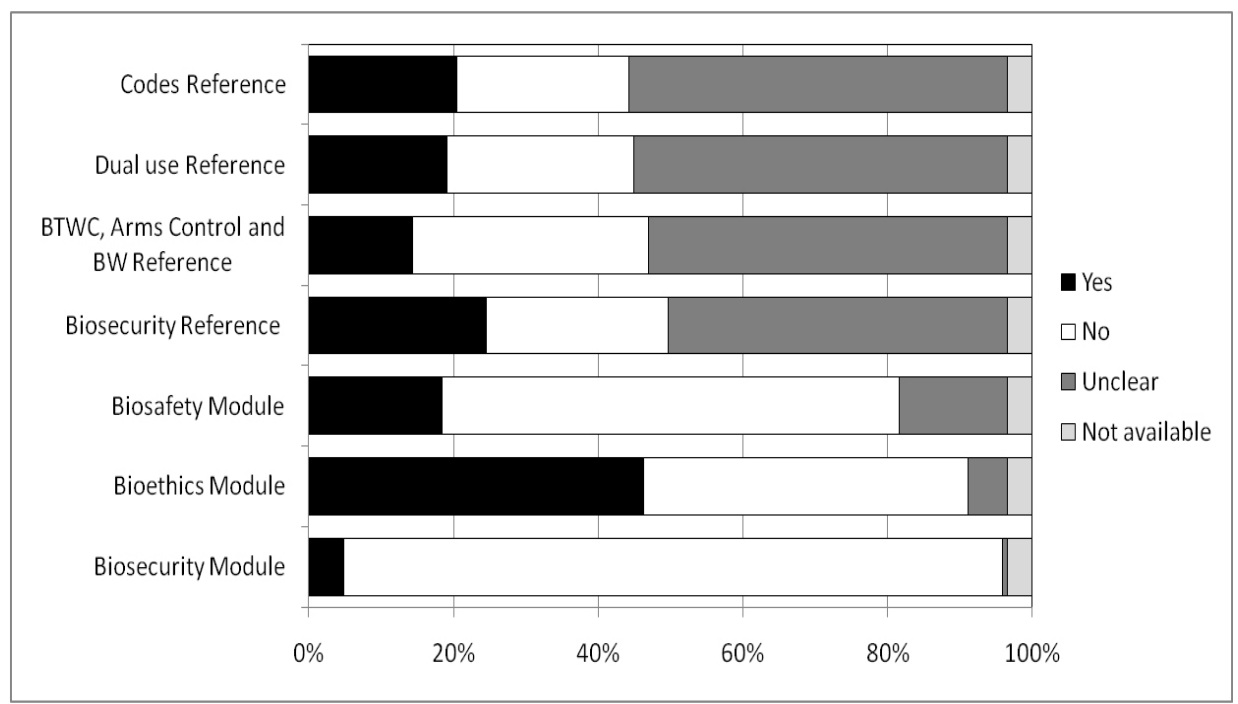

Figure 11: Overview of results from investigation into the biosecurity contents of European life-science and biotechnology courses

This investigation was not intended to produce statistically significant results and suffered a number of problems in determining content with certainty, particularly vis-à-vis 'references' to concepts related to biosecurity. Indeed, what constitutes a 'reference' ranged from one or more dedicated lectures, to a reference during a lecture. Nevertheless, the process provided an illustrative snapshot of the situation in Europe with regards to biosecurity and related components. We found a total of 37 (26 per cent) life-science and biotechnology degree courses from our sample where there was clear evidence of a reference to biosecurity; 22 (15 per cent) courses mentioned biological weapons, the BTWC or other arms-control agreements; in 29 (20 per cent) courses there was clear reference to the dual-use issue and finally, in 31 (22 per cent) courses there was a reference to codes of conduct, practice or ethics.

In terms of generating an understanding of attitudes to biosecurity education, responses to questions regarding whether biosecurity was perceived as important largely fell into three categories, with the majority falling in the first two. In the first category were a number of examples where curricula clearly didn't offer a specific module in their syllabi, or respondents felt the topic was irrelevant. Thus, for example, it was suggested 'we do not teach anything to do with the BTWC. I'm not sure if teaching such material on the BTWC would be helpful to our students unless they went into the field'. In the second category were those that felt the topics were relevant but were constrained by practical factors. Therefore, for example, one participant noted 'the main reason [for not having a specific biosecurity module] being the limited time we have to expose our students to science \& society issues'. Finally, there were those that were 
clearly interested in the inclusion of this material as a topic of discussion; for example, one participant stated, 'I will take profit of your enquiry and integrate these issues in the introductory course starting with this year'.

In addition to the collection of data to determine the extent of biosecurity education in Europe, the process was useful in constructing the collaborative network through the identification of, and engagement with, individuals from various disciplines that were interested in advancing education on these subjects. This process was to some extent confirmed through two workshops held in Como in 2008 and 2009 titled 'Fostering the Biosecurity Norm: An Educational Module for Life-science Students' and 'Biosecurity, Biosafety and Dual-use Risks. Trends, Challenges and Innovative Solutions', respectively, which brought together life-science educators and security experts to discuss these topics. The process of conducting a gap analysis had a clear secondary value in building a biosecurity education network and providing a means through which the target audience could be reached and their attention and active engagement secured.

\section{Building a Biosecurity Education Network}

Indeed, the development of an informal collaborative network of professional actors interested in biosecurity or dual-use education has proved a useful model through which the process of framing and implementing education can be advanced taking into consideration input from a range of actors. As such, this remains one possible practical response to the question posed by Rappert on how audiences and practising scientists can be reached. Camarinha-Matos and Afsarmanesh have defined a collaborative network as being 'constituted by a variety of entities (for example, organisations and people) that are largely autonomous, geographically distributed, and heterogeneous in terms of their operating environment, culture, social capital, and goals. Nevertheless, these entities collaborate to better achieve common or compatible goals.' ${ }^{2}$

Such a model is certainly consistent with the collaborative network established in the LNCV-BDRC project, which consists of a number of independent academic departments and research organisations covering a range of disciplines (life sciences, biotechnology, bioethics, law, political science, security studies and international relations) in various countries in Europe (including Sweden, Italy, Spain, the Netherlands, the UK, Portugal and Germany), and which have in a variety of ways provided input from different perspectives that cumulatively contributed to the advancement of the network and progress in the objectives.

2 Camarinha-Matos, L. M. and Afsarmanesh, H. 2004, 'Collaborative networks: a new scientific discipline', Journal of Intelligent Manufacturing, vol. 16, 2005, available: http://www.uninova.pt/ cam/Papers/ ColNetsJIM.pdf. 
In this regard, the collaborative network can be seen as facilitating both the process of building bridges between different communities and academic disciplines and enhancing the quality of material and the implementation of lectures. Contributions to the legal aspect of biosecurity education have been made, for example, through engagement with the Institute for International Legal Studies at the National Research Council (ISGI-CNR) in Italy and the Department of Penal Law at the University of Granada in Spain. Cooperation with legal experts was particularly fruitful and led to the distillation of domestic legal references developed by a number of countries into accessible material more readily usable by science students and lecturers. More ethically and philosophically orientated consideration has also been advanced through engagement with the Department of Philosophy at Delft University of Technology in the Netherlands. Discussions with scientists and life-science lecturers within the network, such as those in the National Council of Biologists of Italian Universities, have been particularly useful for enhancing the quality and implementation of material. Firstly, they have provided feedback and advice on the optimisation of both teaching material and the delivery of lecturers to specific audiences; and, secondly, they have advised us on how implementation challenges can best be overcome.

\section{Implementation Tests}

Using nodes within the network developed through the survey and the Educational Module Resource elaborated upon by Whitby and Dando in another chapter in this volume, the LNCV-BDRC project has begun the process of testing material in several universities across Europe. This process was facilitated by contacts in the network who agreed to promote lectures or seminars organised by local professors, using agreed elements of material (summarised below in Table 3), as the basis for implementation tests tailored to their local contexts.

In some cases, implementation tests were conducted independently by lifescience educators who developed their own lectures from material developed for the EMR and translated this into the local language where necessary. This suggests that in some cases the implementation of this approach to biosecurity education may merely require directing individuals to structured and accessible educational materials that can be used as a solid base for lectures which necessarily move beyond a scientific focus into broader social, economic and ethical territory. In other cases, network members began a process of engagement with the authors in which components of lecture courses were negotiated. In yet other cases, LNCV-BDRC project officers conducted lectures that were agreed with professors and tailored in time, content and delivery to suit the local audience. 
Education and Ethics in the Life Sciences

Table 3: Summary of material in the EMR

\begin{tabular}{|c|c|}
\hline $\begin{array}{l}\text { Biological Weapons, Biowarfare and Bioterror } \\
\text { - Biological Warfare from Antiquity to World War II } \\
\text { - Biological Warfare in World War II } \\
\text { - Biological Warfare During the Cold War } \\
\text { - Assimilation of BW in Military Programmes and } \\
\text { Calculation of Effects } \\
\text { - Bioterrorism }\end{array}$ & $\begin{array}{l}\text { The Dual-use Risks and the } \\
\text { Responsibilities of Scientists } \\
\text { - BW, Biosecurity and Ethics } \\
\text { - Possible Hostile Applications } \\
\text { of New Technologies } \\
\text { - Hostile Application of Biology } \\
\text { is Prohibited } \\
\text { - Dual-Use: The Fink Report } \\
\text { - Dual-Use: Examples } \\
\text { - The Lemon-Relman Report } \\
\text { - Weapons Targeted at the } \\
\text { Nervous System } \\
\text { - Possible Regulations of the } \\
\text { Life Sciences? }\end{array}$ \\
\hline $\begin{array}{l}\text { The International Prohibition Regime and Implementation } \\
\text { - International Legal Agreements } \\
\text { - Development of the BTWC: } 1980-2008 \\
\text { - The First Intercessional Process: the } 2005 \text { BTWC } \\
\text { Meetings on Codes of Conduct } \\
\text { - The BTWC Second Intercessional Process 2007-11 } \\
\text { - International Customary Law } \\
\text { - United Nations Security Council Resolution } 1540 \\
\text { - EU Legislation and Directives on Export Controls } \\
\text { - National Legislation }\end{array}$ & $\begin{array}{l}\text { The Web of Prevention } \\
\text { - Building an Effective Web of } \\
\text { Prevention }\end{array}$ \\
\hline
\end{tabular}

In all cases there was not sufficient time to cover every component part of the EMR (which is intended as a comprehensive resource), nor was it necessarily important for undergraduate students in different scientific disciplines of relevance to learn all the information outlined in Table 3. However, it was important to ensure that the key elements were covered. In some cases this required a degree of discussion and content negotiation with network members 
where implementation tests were taking place. This was particularly important for engagement with individuals with scientific backgrounds who were more interested in focusing on examples of dual-use research.

This issue is significant in relation to the question posed in the introduction as to how to secure audience attention and active engagement. Three key areas are noteworthy in this regard. Firstly, it is apparent that an extensive section on the history of offensive biological weapons was perceived as unwelcome and unnecessary. Such a response is understandable in courses focused on science. However, the presentation of specific case studies can provide a stimulating discussion, but also an overview of the history of offensive biological-weapons programmes, which remains important in dispelling the myths amongst some in the scientific community that biological weapons have either not been used in warfare, or have been the preserve of other, 'bad' scientists. Secondly, some commentators were reluctant to include significant discussion on either armscontrol measures or legislation on the grounds that this may be irrelevant, boring, or incomprehensible to science students. Whilst recognising such concerns, it is contended that a basic understanding of the legal and regulatory measures affecting scientists remains an essential component of biosecurity education because they can have a direct effect on their future activities.

Finally, in addition to the shortening of material to comply with the requirements of lecturers, it is apparent there is a need to include material which explicitly acknowledges the positive aspects of science, in order to both avoid the project being seen as in any way 'anti-scientific' and to avoid 'scaring' students away from science. On this basis, in more recent implementation tests we have been very clear that any security concerns need to be kept in perspective and the vast array of positive benefits of science in responding to societal challenges in the sphere of, inter alia, health, energy, food security and economic development are acknowledged at the beginning of the lectures. Indeed, for many in the scientific community, this is an essential component to avoid dissuading or deterring individuals away from the life sciences and prevent the appearance of being somehow anti-scientific.

\section{Implementation Test Results}

As part of the implementation test process we also attempted to evaluate the success of our activities. In the short term, there are a number of means of measuring the success of a lecture in raising awareness. These include pre- and post-lecture surveys (which could simply be done through raising hands), examination questions and seminar discussion-group demonstrations of understanding. In this project we opted to primarily use post-lecture surveys. 
Using this approach it has been possible to determine the success of an individual lecture and, indeed, the results from implementation tests conducted with network members. These appear positive, with a significant percentage of participants indicating that they thought their knowledge of biosecurity and other related topics had been improved, as illustrated in Figure 12.

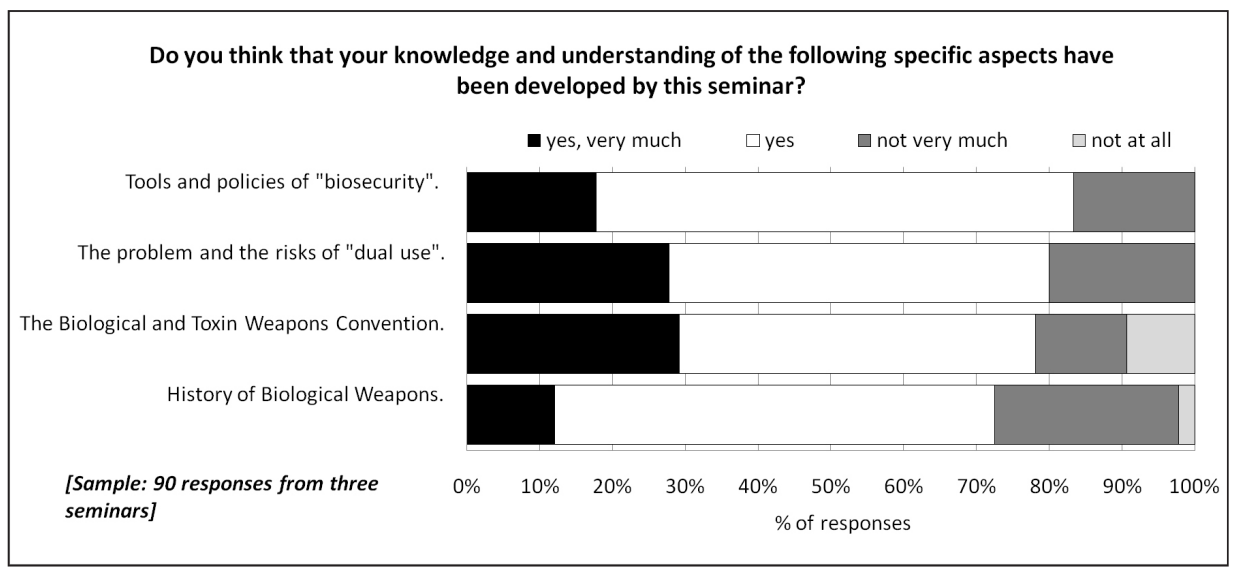

Figure 12: Responses to post-lecture questionnaire

It was also apparent that a significant majority of participants felt the lectures were both interesting and important, with 99 per cent appreciating the seminars and agreeing that raising awareness of biosecurity-related issues should be promoted among students, something evident in Figure 13.

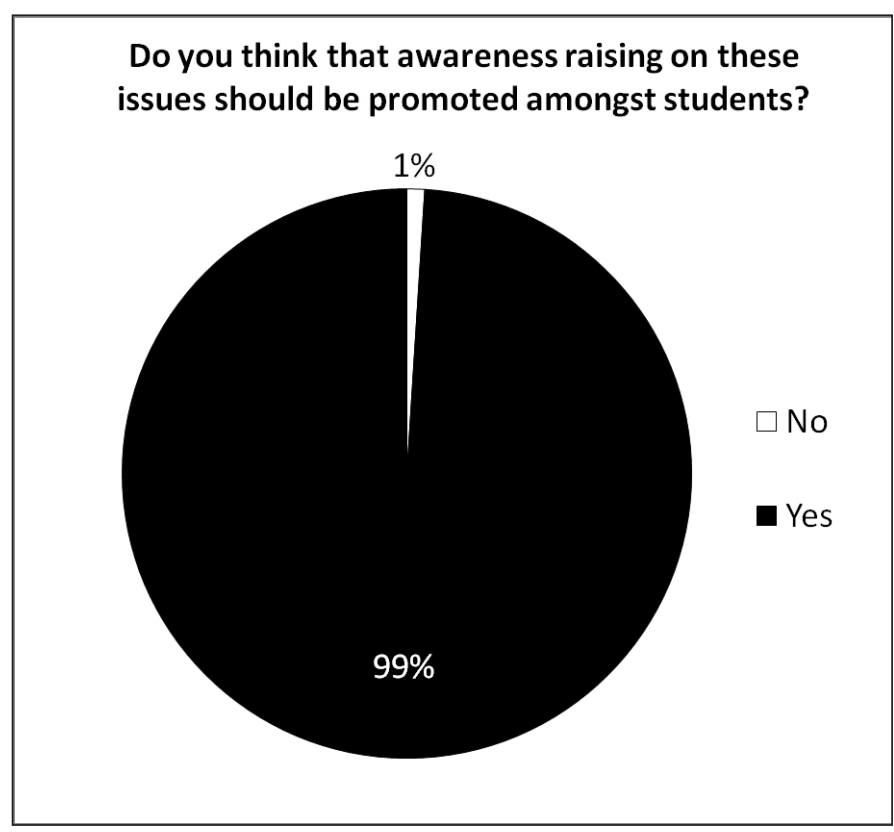

Figure 13: Responses to post-lecture questionnaire II 
Such support for the promotion of biosecurity education is important to feed into a second test of success, that of sustainability. Sustainability is important in meeting the objective of building a culture of awareness which passes material on to subsequent generations and there are a number of metrics that can be used individually or in combination to test sustainability effectively. Examples include the following:

- post-lecture discussions with staff and students

- follow-up questionnaires

- requests for information on subsequent activity

- monitoring of website providing educational material related to biosecurity to determine the frequency and geographic location of downloaded material.

Based on these criteria, we can claim a degree of success in that network contacts in several cases have intimated that, given the interest raised and the available reference materials, they intend to both recommend and replicate lectures in future academic years. Accordingly, it has been possible to determine success in the immediate impact of generating interest and the potential future sustainability of lectures.

\section{Measuring Prevention}

However, in the longer term determining the success of preventing scientists (regardless of a terrorist or state affiliation) from contributing to the development of biological weapons is much more difficult. Also, although there may be measures that can be used to assess the long-term impact of biosecurity education, greater consideration is required into how to develop metrics that are sufficiently robust to demonstrate success or failure, yet simple enough to be useful to the policy community. Indeed, it is not enough to simply count the number of students taught. The National Academies of Science have suggested that experience with other Cooperative Threat Reduction (CTR) activities in the US indicated "hard "scorecard" metrics, often very quantitative in nature, are not always going to be adequate measures of a programme's success'. ${ }^{3}$ This is certain to be the case in relation to biosecurity and dual-use education, where demonstrating the effect in the long term to some extent requires proving a negative without confusing causations.

3 National Academies of Science 2009, Global security engagement: A new model for Cooperative Threat Reduction, available: http://www.nap.edu/catalog/12583.html 


\section{Lessons Learned}

Based on the authors' experience with biosecurity and dual-use education, a number of key practical lessons have emerged. These are not necessarily applicable to other regions and future activities are likely to need tailoring to the specific location. Nonetheless, they may prove useful in facilitating future activities.

One of the principle lessons learned over the course of building the network and conducting implementation tests is the need to carefully frame both engagement and presentation of material. The perception of biosecurity education as irrelevant or less relevant amongst many (not all) in the scientific community, necessitates that the rationale for including it needs to be clearly and objectively articulated. This applies both at university level, where it is essential to ensure science and scientist are not presented as part of the problem but part of the solution, but also to any activity related to other intervention points that could be conducted. Indeed, the need to frame the issue carefully is likely to be even more acute in relation to national academies and authors.

In practical terms, discussion with lecturers and course coordinators in the European context indicates there is only limited space within the degree course syllabi. Thus, space for extensive historical material and detailed information on conventions and regulations is likely to be difficult to integrate within the often-small window of opportunity that exists to engage life-science students. As one survey respondent suggested, 'all knowledge is useful. It is a matter of priorities and of limited number of credits/programme [space available]'. Accordingly, if the objective is to reach a broader audience using the bottom-up approach outlined above, compromise will often be necessary.

Objectivity is also very important to students of disciplines such as biology that are founded on positivist logic. Thus it is often advantageous to avoid subjective approaches or, more significantly, 'preaching' to life-science students. This is particularly acute in relation to more ethically orientated issues such as the morality of using disease in warfare and our experience concurs with the sentiments of Rappert expressed in Chapter 1 that 'resistance can be intense when some try to tell others what they should think'. Finally, there is no onesize-fits-all approach to achieving biosecurity education through a bottom-up approach. A degree of flexibility in the presentation of materials and content is also likely to be important in the future and the model used in the European context is unlikely to be directly applicable to other contexts. 


\section{Addressing Other Intervention Points}

Although much of the current attention to education is focused on universitylevel education, this is not the only possible implementation point through which biosecurity education can be progressed and it would be remiss not to address the range of others. In their chapter, Whitby and Dando discuss the role of funders of scientific research in encouraging consideration of the so called dual-use issue. If education is considered an important element of what has been termed the 'web of prevention', "there are a number of further intervention points that can and should be addressed which would be mutually reinforcing, examples of which are evident in Figure 14. Indeed, based on a case study produced by Revill, it suggests that if calls for biosecurity education are converted into effective action there are a number of additional intervention points which are particularly important to engage. These include academies of science, authors of scientific textbooks, the biotechnology industry, and possibly schools. ${ }^{5}$ Each one of these points is addressed in turn in the following sections.

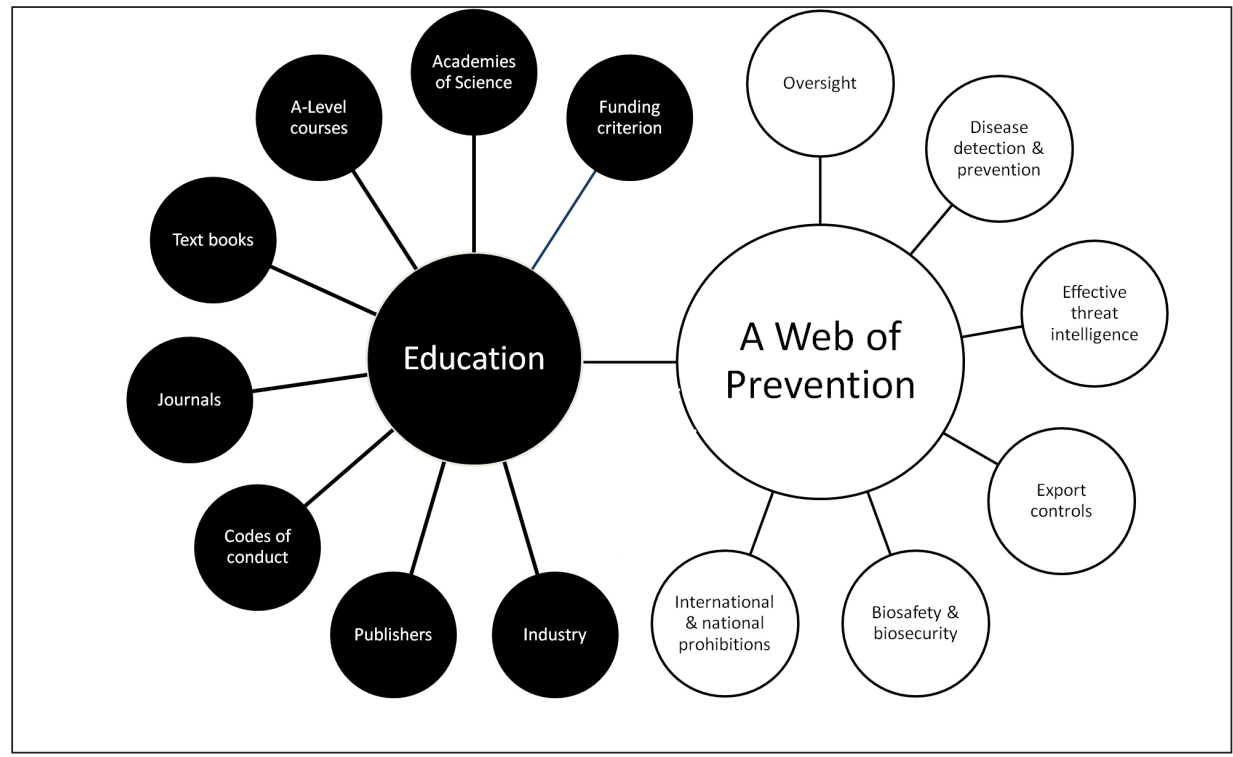

Figure 14: The role of education, and sub activities related to education, within the web of prevention

\footnotetext{
4 Rappert, B. and McLeish, C. (eds), A Web of prevention: Biological weapons, life sciences and the future governance of research, London: Earthscan: 51-65, available: http://people.exeter.ac.uk/br201/Research/ Publications/Chapter\%203.pdf [viewed 1 November 2009].

5 Revill, J. 2009, 'Biosecurity and bioethics education: A case study of the UK context', Research Report for the Wellcome Trust Project on 'Building a Sustainable Capacity in Dual-use Bioethics', December 2009, available: http://www.brad.ac.uk/acad/sbtwc/dube/publications/BioseBioethicsUK.pdf.
} 


\section{Academies of Science}

Academies of science and 'champions' ${ }^{6}$ within the scientific community certainly represent key means of promulgating biosecurity education internally within the community of life scientists, a process that is likely to be considerably more influential than attempts by individuals exogenous to this community. Already there are examples of how individual champions can generate awareness around certain issues, as former UN Secretary General Kofi Annan stated of Joseph Rotblat, 'Mr Rotblat went from working on the nuclear bomb to founding the Pugwash conference, and continued for the rest of his days to champion the principle of scientists taking responsibility for their inventions'. ${ }^{7}$ However, in the current climate it seems the life sciences lack such champions and although some national academies of science have demonstrated interest in the area there is insufficient attention placed on this issue to trigger significant activity.

\section{Scientific Literature and Authors}

It is also apparent, certainly in the UK, that much (not all) of life-science literature tends to omit discussions on biosecurity. This is understandable given the relatively new nature of these terms in the framework of international security discussions, and the perceived irrelevance of the topic to science and scientist. Similarly, although there is often material on biological weapons, this is within the context of agent characteristics and from a scientific perspective that is devoid of material on the illegality of the use of biology as a weapon. This is also logical given the target audience and the intended purpose of life-science textbooks. Nonetheless, a modest extension could serve to engender further debate on prohibitions and regulations that could raise awareness. There are grounds for optimism in this regard and discussion with textbook authors revealed that some might be willing to include a reference to these issues in future volumes. Certainly, Professor Robert Bauman, author of Microbiology with Disease Taxonomy has stated in discussion with Revill that he plans on 'expanding the content of the section on biological weapons to indicate the illegality of these weapons', believing it 'wise to enhance greater understanding of this important issue'.

6 Certainly the idea of 'champions' was voiced at the National Academies of Science [US] meeting on 'Education on Dual Use Issues in the Life Sciences', held on 16-18 November 2009, Warsaw, Poland.

7 Annan, K. 2005, 'Annan regrets death of Nobel Peace Laureate and disarmament advocate Joseph Rotblat', Pugwash Collection of Tributes to Joseph Rotblat, available: http://www.pugwash.org/publication/obits/obitrotblat-tributes.htm. 


\section{The Biotechnology Industry}

The role of industry in education could also prove a valuable intervention point for biosecurity education. Like academies of science, the biotechnology industry is likely to have greater sway upon science students than are social scientists or policymakers who, in some cases, are already perceived as overly burdening and constraining scientific research. Moreover, such a process could present benefits and opportunities for corporations to demonstrate a commitment to social and ethical responsibility.

\section{Conclusions: One Approach to the Implementation of Education}

In his introduction to this volume, Rappert posited a number of questions that need to be considered in the process of moving biosecurity education from an aspiration to a concrete activity. One such question was: How can audiences of practising scientists or other practitioners be reached and how can their attention and active engagement be secured? Although it would be inappropriate to claim ownership of any definitive response, it is apparent that the process undertaken through the LNCV-BDRC study provides a useful overview of the extent of education, but also an understanding of the attitudes of life-science educators and a collection of contacts necessary to construct a collaborative network. This can have practical value as a vehicle to conduct implementation tests and convey ownership of the process of biosecurity education to life scientists. Although the efficacy of this approach in the context of other cultures remains contestable and further research and adaptation may be required to tailor education to the local context, in Europe and other Western academic environments this network process is one constructive bottom-up model through which an audience can be reached and their attention and active engagement secured. Indeed, the network has proved useful in conducting implementation tests in academic departments around Europe and learning to tailor the material and delivery of the EMR.

It has further demonstrated there is no one-size-fits-all approach to education even within the framework of European universities; and although a core module resource is a useful basis for implementation, the context needs to be taken into account. Indeed, based on lessons learned over the course of this project, it is essential to recognise the various priorities of different communities of practice and ensure that material is delivered objectively in a manner that avoids preaching and balances the potentially harmful effects of life-science research with the vast array of positive benefits generated by scientific research. 
This is likely to be a greater challenge in cases where the model was applied more broadly within other contexts, particularly those where scientific research assumes a much higher priority than security of scientific research.

Finally, the implementation of biosecurity at university level is only one element of a number of measures that could be taken to build a 'culture of responsibility'. Other intervention points, such as academies of science and 'champions', the biotechnology industry, and scientific authors, all have a significant role to play advancing biosecurity education. As many of the questions relating to the 'who, what and how of education' will ultimately be answered by the scientific community, significant input and engagement with these other intervention points will be essential in the long term. 


\title{
Chapter 10: Biosecurity Awareness- raising and Education for Life Scientists: What Should be Done Now?
}

\author{
SIMON WHITBY AND MALCOLM DANDO
}

\section{Introduction: Awareness}

The 1975 Biological and Toxin Weapons Convention (BTWC) added to the ban on the use of biological weapons embodied in the 1925 Geneva Protocol by what was termed the 'General Purpose Criterion' of Article 1. This stated that: ${ }^{1}$

Each State Party to this Convention undertakes never in any circumstances to develop, produce, stockpile or otherwise acquire or retain:

1. Microbial or other biological agents, or toxins whatever their origin or method of production, of types and in quantities that have no justification for prophylactic, protective or other peaceful purposes...

Thus, peaceful uses of the modern life sciences are fully protected, but there is an all-encompassing prohibition of non-peaceful development, production, stockpiling, acquisition or retention of microbial or other biological agents or toxins (and toxins here are understood to cover all mid-spectrum agents such as bioregulators).

As early as the Second Five-Yearly Review Conference of the BTWC in 1986, the 'States Parties' 2 recognised the importance of the awareness and education of life scientists in regard to the Convention. In the Final Declaration of

\footnotetext{
1 For the text of the Convention, see: www.opbw.org.

2 The term 'States Parties' refers to the membership of the Convention. The Biological Weapons Convention currently has 163 States Parties and 13 signatories. There are 19 states which have neither signed nor ratified the Convention.
} 
the Conference, States Parties ${ }^{3}$ noted, in relation to Article IV on national implementation measures, that: ${ }^{4}$ 'The Conference notes the importance of ...inclusion in textbooks and in medical, scientific and military educational programmes of information dealing with the prohibition of microbial or other biological agents or toxins and the provisions of the Geneva Protocol.'

Similar statements were subsequently agreed at following Review Conferences. However, during the 1990s, when concerns about Biological Warfare (BW) returned, the attention of diplomats was centred on the problem of how confidence in compliance with the Convention might be improved and there was very little involvement of the civil life-science community. ${ }^{5}$ After the failure of these efforts, when states decided to discuss and promote common understandings on more tractable issues, Australia reported in a 2005 Intercessional Meeting on Codes of Conduct that: ${ }^{6}$

1. Amongst the Australian scientific community, there is a low level of awareness of the risk of the misuse of the biological sciences to assist in the development of biological or chemical weapons. Many scientists working in 'dual-use' areas simply do not consider the possibility that their work could inadvertently assist in a biological or chemical weapons programme...

At the same meeting we reported work carried out with the editor of this volume, Brian Rappert, in which we had interactive seminar discussions with life scientists at 15 UK universities. Analysis of the tape recordings of these seminars led us to conclude that: 'There is little evidence from our seminars that participants: a. regarded bioterrorism or bioweapons as a substantial threat; $b$. considered that developments in life-sciences research contributed to biothreats; c. were aware of the current debates and concerns about dual-use research; or d. were familiar with the BTWC.' 7

In the next year we reported to the Sixth Review Conference on further seminars in several other countries. In regard to the UK seminars we concluded that: 'The results from the remainder of the seminars were consistent with all

3 States Parties refers to States that have both signed and ratified the Biological and Toxin Weapons Convention. (BTWC). The BTWC currently has 163 States Parties and 13 signatories. There are 19 states which have neither signed nor ratified the Convention.

4 Again, for the text of the Final Declaration, see: www.opbw.org.

5 Dando, M. R. 1994, Biological warfare in the 21st century: Biotechnology and the proliferation of biological weapons, London: Brasseys; Dando, M. R. 2002, Preventing biological warfare: The failure of American leadership, Basingstoke: Palgrave.

6 Australia 2005, Raising Awareness: Approaches and Opportunities for Outreach, BWC/MSP/2005? MX/ WP.29, available: www.opbw.org.

7 Dando, M. R. and Rappert, B. 2005, Codes of Conduct for the Life Sciences: Some Insights from UK Academia, Briefing Paper no. 16 (2nd series), University of Bradford, May, available: www.brad.ac.uk/acad/sbtwc. 
of these points [enumerated above]. A particular surprise was that so few of the participants (less that 10 per cent in most groups) had heard of the mousepox experiment that has figured largely in security literature.' 8

There were, of course, some differences in the interactions that we reported in the seminars in the Netherlands, Finland, the US and South Africa but we stated that ' $[D]$ espite such differences between the seminars held in the different countries, the degree of similarity between the responses in the seminars was much more pronounced'. Our subsequent experience of carrying out seminars in 16 different countries with a few thousand life scientists in over 110 different departments has consolidated these findings. ${ }^{9}$ Indeed, we used the seminars more as an awareness-raising mechanism rather than as a means of investigative research into the attitudes of life scientists.

\section{The Education Gap}

These findings demand some serious explanation. Many physicists are clearly aware of the dangers of the misuse of their science and have played important roles, for example, in the Pugwash movement. In the 1980s and 1990s, chemists were also influential in helping to bring negotiations of the Chemical Weapons Convention (CWC) to a successful conclusion, and the International Union of Pure and Applied Chemistry (IUPAC) has contributed major reviews of relevant science and technology to the first two Review Conferences of the CWC. Therefore, it is not unreasonable to ask why practising life scientists are so unaware of the BTWC and the problem of dual use despite increasing attention being given to these issues, for example, by national science academies.

One possible explanation is that life scientists are uninformed of biosecurity issues because they do not feature in their university education. In order to investigate this possibility, in cooperation with the Italian Landau Network Centro-Volta, we carried out an internet survey of a sample of courses in the EU. As detailed further in the chapter by Mancini and Revill, the results were quite startling: 'This research suggested that only three out of 57 Universities identified currently offered some form of specific biosecurity module and in all cases this was optional for students.' ${ }^{10}$ On the other hand, the survey noted:

\footnotetext{
8 Rappert, B., Chevrier, M. I. and Dando, M. R. 2006, In-depth Implementation of the BTWC: Education and Outreach, Review Conference Paper no. 18, University of Bradford, available: www.brad.ac.uk/acad/sbtwc. 9 Rappert, B. 2007, Biotechnology, security and the search for limits: an inquiry into research and methods, Basingstoke: Palgrave; Rappert, B. 2009, Experimental secrets: International security, codes, and the future of research, University Press of America.

10 Mancini, G. and Revill, J. 2008, Fostering the biosecurity norm: Biosecurity education for the next generation of life scientists, London Network, Centro Volta and University of Bradford, available: www. Dual-use bioethics.net.
} 
There is evidence of a considerable number of bioethics modules and nearly half of the degree programmes surveyed evidenced some form of bioethically-focused module. In terms of biosafety modules... roughly one-fifth of life-science degrees in the sample contain a specific dedicated biosafety module although several of these specific modules were optional.

So we found a reasonable number of biosafety modules, a large, and we suspect, increasing number of bioethics modules, and virtually no biosecurity modules.

We attempted to investigate in more detail by looking for any kind of reference to biosecurity issues in the course material. Again the picture was bleak:

Exactly what constitutes a reference varies; however, based on the quantitative data from the investigation, we found a total of 37 lifescience degree courses out of our sample of 142 where there was clear evidence of a reference to biosecurity. Only a minority of the degree courses in the study - a total of 22 out of 142 - made a reference to the BTWC, BW and/or arms control, and a similar number, 29 degree courses, exhibited some reference to the dual-use issue.

When we carried out a similar survey in Japan, and as can be seen from the analysis presented by Minehata and Shinomiya, we found a similar picture. Of 197 life-science degree courses in 62 universities we found only three specific biosecurity modules. ${ }^{11}$

In Japan we took the investigation a stage further by sending out a questionnaire to lecturers asking why biosecurity and dual use was not being taught. Clearly some lecturers did not see these subjects as relevant to their courses, but others certainly did. Where people thought the topics relevant but did not teach them, the reasons cited were a lack of expertise and access to necessary resources, and a lack of space on a very crowded timetable in the modern life sciences.

\section{Correcting the Deficiency}

Correcting this deficiency in education- and awareness-levels of life scientists will be a massive task that will require action by a range of constituencies involved in life-science education including, inter alia, governments, bodies

11 Minehata, M. and Shinomiya, N. 2009, Dual-use education in life science degree courses at universities in Japan, National Defence Medical College of Japan and Bradford University, available: www.dual-use bioethics. net. 
responsible for the administration of standards in higher education, funders of life-science education, civil society groups and non-governmental organisations involved in the production of educational material, and teachers and trainers. ${ }^{12}$

As is evidenced by the convergence of ethics and medicine in the area of biomedical ethics, ${ }^{13}$ the consideration of moral dilemmas is not new in lifescience research. However, current concern about dual use ${ }^{14}$ - where science findings can be used for malign as well as benign purposes - arises from a new range of security threats. These include the changing nature of warfare, the possibility of new forms of mass-casualty terrorism, a discernable commitment by States Parties to the BTWC to address these threats through seeking to improve awareness and education amongst life scientists, reviews of scientific oversight regarding dual-use research performed by national scientific academies ${ }^{15}$ (particularly in the US), and a genomic and biotechnology revolution in life science with the rapid and worldwide spread of advanced science and technology. Thus concerns about dual use are being discussed in the context of a distinctly new phenomenon - namely, a convergence between security concerns and the practice of life scientists in what might be termed a novel biosecurity problem.

The term 'biosecurity' has been used in different ways in different contexts. We should, therefore, be very clear about usage of the term here. In our view the threat spectrum ranges from natural disease through to inadvertently caused disease. We deal with natural disease by public-health measures and inadvertently caused disease is restricted by 'biosafety' - good laboratory practice. The concept of laboratory biosecurity has also arisen to ensure that dangerous materials are kept secure from those with malign intent. We see laboratory biosecurity as part of biosecurity, but for us the term has a much wider meaning related to the concept of a web of preventative policies centred on the prohibition of the misuse of the life sciences embodied in the General Purpose Criterion of the BTWC. Thus biosecurity is the objective of the whole range of policies, such as export controls, biodefence and national implementation of the Convention, that minimise the possibility that the life sciences will be misused for hostile purposes. Within that range of policies there is, in our opinion, a role for practising life scientists in being aware that the materials, technologies and knowledge they produce may be misused and for contributing their expertise to the development and maintenance of preventative policies.

\footnotetext{
12 Dando, M. R. 2009, Dual-use Education for Life Scientists, Disarmament Forum, vol. 1, pp. 41-4.

13 Jonsen, A. R. 1998, The birth of bioethics, lst edition, USA: Oxford University Press.

14 Atlas, R. and Dando, M. 2006, 'The dual-use dilemma for the life sciences: Perspectives, conundrums, and global solutions', Biosecurity and Bioterrorism: Biodefense Strategy, Practice, and Science, vol. 4, pp. 276-86.

15 The US National Academy of Sciences' (Fink Committee) classification of seven classes of experiment sought to illustrate the types of endeavour that would require careful review by informed experts.
} 
In order to begin building capacity in biosecurity education, a new range of creative and innovative interventions are required. As set out in the following argument, it is seen that biosecurity education can be easily accommodated by current standards in higher education in the UK. In the US, recommendations for the adoption by federally funded institutions of biosecurity education have already been set out.

\section{Scope for Biosecurity Education in the UK and US}

Whilst ethical consideration of the implications of dual-use science and technology is conspicuously absent from the vast majority of curricula in UK higher education, in the US, and indeed worldwide, it is apparent that a codified response through the development of new guidelines and policies that reflect biosecurity concerns will not necessarily be required within UK higher education. In order to satisfy its statutory obligation to ensure that publicly funded teaching provision is of a high standard, the UK's Higher Education Funding Council (HEFCE) ${ }^{16}$ contracts the Quality Assurance Agency (QAA) ${ }^{17}$ to 'devise and implement quality- assurance methods' and is responsible for the conduct of audit and review of teaching quality in both higher and further education. Although not a national curriculum that sets standards in UK higher and further education, the Subject Benchmark Statements produced by the Quality Assurance Agency ${ }^{18}$ require the inclusion of an ethical dimension in all undergraduate bioscience programmes. These are largely aspirational; however, they 'set out expectations about standards of degrees in a range of subject areas. They describe what gives a discipline its coherence and identity, and define what can be expected of a graduate in terms of the abilities and skills needed to develop understanding or competence in the subject.' ${ }^{19}$

The 2002 QAA bioscience benchmark statements made a number of references to 'ethical' aspects of this subject-area, including the following requirements:

Students should expect to be confronted by some of the scientific, moral and ethical questions raised by their study discipline, to consider viewpoints other than their own, and to engage in critical assessment and intellectual argument. ${ }^{20}$

16 http://www.hefce.ac.uk/Learning/qual/qaa.asp.

17 http://www.qaa.ac.uk/academicinfrastructure/benchmark/default.asp.

18 UK Quality Assurance Agency (QAA) 2002 \& 2007, Honours Degree Subject Benchmark Statements, Bioscience, available: http://www.qaa.ac.uk/academicinfrastructure/benchmark/statements/Biosciences07. pdf.

19 Ibid.

20 Ibid. 
Recognising the moral and ethical issues of investigations and appreciating the need for ethical standards and professional codes of conduct. ${ }^{21}$

All students should: Have some understanding of ethical issues and the impact on society of advances in the biosciences. ${ }^{22}$

Good students should: Be able to construct reasoned arguments to support their position on the ethical and social impact of advances in the biosciences. ${ }^{23}$

Honours Degree Subject Benchmark Statements were re-stated by QAA again in 2007, where the Subject Benchmark Statement for Biosciences again reiterated the importance of the inclusion of an ethical dimension in undergraduate programmes. Whilst ethics teaching forms an important component in many bioscience courses and courses address a range of ethically related issues, ethics in bioscience or bioethics education could easily be extended to accommodate and incorporate the ethical concerns of biosecurity education. Institutional audits of teaching-quality assessment by the QAA strengthen incentives to extend teaching in ethics into the area of biosecurity.

Further to this, a report ${ }^{24}$ published in December 2008 by the US National Advisory Board for Biosecurity (NSABB) sets out a Strategic Plan for Outreach and Education on Dual-Use Research Issues. As specified in a related contribution to this book, this strategy envisages the implementation of a series of recommendations on 'the development of programmes for outreach, education, and training on dual-use research issues for all scientists and laboratory workers at federally funded institutions in the US'.

\section{Funders of Science}

Increasingly, recipients of research funding must be willing to comply with requirements set out by funders of science that are intended to ensure bioscience-research activities are in full compliance with guidance on ethics. Indeed, in the case of some funders of bioscience, reference to dual-use research is now explicit.

24 National Science Advisory Board for Biosecurity 2008, Strategic plan for outreach and education on dual use research issues, December, Washington, DC.
} 
As outlined in its position statement ${ }^{25}$ on Research Ethics, the UK Biotechnology and Bioscience Research Council (BBSRC) states that it has:

a responsibility to ensure that its funds are used ethically and responsibly. Potential applicants should consider whether their work is likely to give rise to societal concerns about the purpose of the research, or includes any social or ethical issues regarding its conduct or potential outcomes (for example, relevance to development of biological weapons; products and processes that might be used in social discrimination), or other aspects of potential public concern.

As stated in its Terms and Conditions ${ }^{26}$ for Research Council Grants, according to the Research Councils UK (RCUK), recipients of its funding are responsible for ensuring that:

...ethical issues relating to the research project are identified and brought to the attention of the relevant approval or regulatory body. Approval to undertake the research must be granted before any work requiring approval begins. Ethical issues should be interpreted broadly and may encompass, among other things, relevant codes of practice, the involvement of human participants, tissue or data in research, the use of animals, research that may result in damage to the environment and the use of sensitive economic, social or personal data.

The UK Medical Research Council (MRC) ${ }^{27}$ sets out detailed guidance on ethics that addresses a broad range of areas including 'clinical research governance', 'global bioethics' and 'good research practice'. Additionally, the MRC Position Statement on Bioterrorism and Biomedical Research recognises the 'dual-use nature of life science and the importance in funding research of due consideration of ethical dilemmas presented by research'.

Adopting a similar approach, the Wellcome Trust specifies the importance of appropriate processes existing at institutional, national and international levels for the review and oversight of dual-use research. In its Position Statement on Bioterrorism and Biomedical Research, the Wellcome Trust ${ }^{28}$ cites the US National Academy of Sciences' (Fink Committee) classification of seven classes of experiment to illustrate the types of endeavour that would require careful review by informed experts. The experiments this committee specified are those that would:

- demonstrate how to render a vaccine ineffective

25 http://www.bbsrc.ac.uk/funding/apply/grants_guide.pdf.

26 http://www.rcuk.ac.uk/cmsweb/downloads/rcuk/documents/tcfec.pdf.

27 http://www.mrc.ac.uk/Ourresearch/Ethicsresearchguidance/index.htm.

28 http://www.wellcome.ac.uk/About-us/Policy/Policy-and-position-statements/WTD002767.htm. 
- confer resistance to therapeutically useful antibiotics or antiviral agents

- enhance the virulence of a pathogen, or render a non-pathogen virulent

- increase transmissibility of a pathogen

- alter the host range of a pathogen

- enable the evasion of diagnostic and detection modalities

- enable the weaponisation of a biological agent or toxin.

Improved grant-application procedures, more stringent reporting requirements and management, and oversight of the grants by funders will help ensure that grantees live up to stipulated ethical obligations and efficient and effective implementation of such requirements will facilitate the development of best practice in the financing of life-science research. Given the low levels of awareness amongst life scientists of biosecurity issues it is not surprising that progress has been slow in the implementation of measures such as effective training programmes to address the issue. However, it can be expected that these measures will be implemented in coming years: the question is, how quickly and how well?

\section{Civil Society Groups and Non-Governmental Organisations}

In the meantime, there does seem a potential role for civil society in providing models of what might be done to close the gap most effectively in the shortest timeframe. This is what we have been attempting to do over the last few years in developing a Dual-use Biosecurity Education Module Resource (EMR). The NSABB report 'Strategic Plan for Outreach and Education on Dual Use Research Issues' ${ }^{29}$ mentioned previously considers what needs to be done in some detail. In its view, developing a strategic plan requires: 'First and foremost, the target audience must be identified and assessed as to their level of understanding of the issues since this will guide educational strategies...[Then]...messages should be tailored to specific target-audiences. Key points must be identified and specifically crafted.' And because there are so many different possible methods of communication, 'it is important to select those methods that will most effectively reach the intended audiences'.

Therefore, when we applied a similar method of analysis to our work, it was clear that our intended target audience - university-level lecturers and students - did not have a high level of awareness of biosecurity and dual-use issues. Furthermore, given the prevalence of the use of the internet in universities it

29 National Science Advisory Board for Biosecurity 2008, op. cit. 
was clear that providing information on the web was by far the most efficient and effective way forward. However, given the pressure on the timetable we thought it unwise to design a one-size-fits-all educational module and decided to design an EMR that could be used by different lecturers to fit relevant parts into their own courses.

Our thinking was also much influenced by the developing consensus about education of life scientists that developed at the 2008 BTWC Intercessional Meetings. The final report of these meetings states: ${ }^{30}$

26. States Parties recognised the importance of ensuring that those working in the biological sciences are aware of their obligations under the Convention and relevant national legislation and guidelines, have a clear understanding of the content, purpose and foreseeable...security consequences of their activities, and are encouraged to take an active role in addressing the threats posed by potential misuse of biological agents and toxins as weapons, including bioterrorism.

This paragraph of the report then continues, significantly: 'States Parties noted that formal requirements for seminars, modules or courses, including possible mandatory components, in relevant scientific and engineering training programmes and continuing professional education could assist in raising awareness and in implementing the Convention.'

In the paragraph that followed, States Parties set out what they agreed would be of value in such programmes:

(i) Explaining the risks associated with the potential misuse of the biological sciences and biotechnology

(ii) Covering the moral and ethical obligations incumbent on those using the biological sciences

(iii) Providing guidance on the types of activities which could be contrary to the aims of the Convention and relevant national laws and regulations and international law

(iv) Being supported by accessible teaching materials, train-the-trainer programmes, seminars, workshops, publications, and audio-visual materials

30 Meeting of the States Parties to the Convention on the Prohibition of the Development, Protection and stockpiling of Bacteriological [Biological] and Toxins Weapons and on their Destruction (2008) Report of the meeting of States Parties, BWC/MSP/2008/5, United Nations, Geneva, 10 December. 
(v) Addressing leading scientists and those with responsibility for oversight of research or for evaluation of projects or publications at a senior level, as well as future generations of scientists, with the aim of building a culture of responsibility

(vi) Being integrated into existing efforts at the international, regional and national levels.

Our idea for the EMR was to capture as many of these ideas as possible based on the concept of having a web of integrated preventative policies that together would persuade everyone thinking of breaking the prohibition that the costs would far outweigh the benefits. ${ }^{31}$ However, in work with colleagues at Japan's National Defence Medical College on designing and testing the EMR (under British Council Funding) it became clear that we needed to start the lecture series with material that could be readily grasped by life scientists.

Thus the EMR consists of 21 lectures, each with 20 PowerPoint slides and notes for the lecturer, and direct links to the references used via the web. Each lecture also has some suggested essay questions and the EMR has an introduction to all the material for lecturers and a small number of Briefing Papers cover material that would be less familiar to life scientists. Several lectures are also duplicated, with material in the second set being more scientifically orientated.

Therefore, our EMR is designed in five parts as follows:

Outline of the EMR

\section{A. Introduction and Overview}

\section{Lecture 1}

B. The Threat of Biological Warfare and Biological Terrorism and the International Prohibition Regime

Lectures $2-10$

C. The Dual-Use Dilemma and the Responsibilities of Scientists

Lectures 11-18

D. National Implementation of the BTWC

Lectures 19-20

E. Building an Effective Web of Prevention

31 Rappert, B. and McLeish, C. 2007, A Web of prevention: Biological weapons, life sciences and the governance of research, London: Earthscan. 
Lecture 21

Thus the first lecture gives a brief overview of the whole of the module resource in order to orientate the user.

The second section takes up the story of the misuse of modern biology after the discovery of the causes of infectious diseases in the late nineteenth century by scientists such as Pasteur and Koch. This history is largely unknown amongst life scientists and forms a basis for consideration of the possible misuse of future advances. In this section we have also introduced modern accounts of the traditional agents such as anthrax, smallpox and botulinum toxins to better engage scientists' interest.

The lectures in Section B are set out as follows:

Section $B$ of the EMR

2. BW from Antiquity to World War I

3. BW from WWI to WWII

4. BW during the Cold War

5. The impact of BW Agents

6. Assimilation of BW in State Programmes

7. International Legal Agreements

8. Strengthening the BTWC 1980-2008

9. The 2003-2005 Intercessional Process

10. The 2007-2010 Intercessional Process

Section B ends by briefly reviewing how the international community has attempted to deal with the threat of the proliferation of biological weapons through the 1925 Geneva Protocol, the 1975 BTWC and the 1997 CWC (given that there is an overlap between the BTWC and CWC in the area of mid-spectrum agents such as toxins and bioregulators).

It can be seen that these lectures begin with a consideration of the history of biological warfare and end with the BTWC recent annual meetings in which scientists have become increasingly involved - at least at the level of national academies and industrial leaders. This then sets the basis for the third section of our module.

The lectures in Section C are set out as follows: 
Section $C$ of the EMR

11. Bioethics methodology

12. Obligations derived from the BTWC

13. The growth of dual-use bioethics

14. Dual-use: The Fink Report

15. Dual-Use: Examples

16. The Lemon-Relman Report

17. Weapons targeted at the nervous system

18. Regulation of life sciences

Although present evidence strongly suggests there is little biosecurity or dualuse content in university life-science modules dealing with bioethics, it is our belief this is probably the best place to focus on these issues. Life scientists are becoming familiar with the ethical problems that new research brings up, and the teaching of bioethics is growing in universities. Our view is that biosecurity and dual-use issues are best presented to life scientists in the context of the moral and ethical implications of research (see item (ii) in the 2008 report of the BTWC meeting on education above). ${ }^{32}$ Therefore, this section of the module starts with a review of standard bioethical analyses that students are likely to have encountered before, introducing the growing literature on dual-use bioethics. The section then leads to a consideration of the key US National Academics Reports (Fink and Lemon-Relman) that began the closer examination of the dual-use problem from within the scientific community. Some lectures examine classic dual-use experiments such as the mousepox experiment in lecture 15 and the contention by Lemon-Relman that the dual-use problem is far wider than just research in microbiology, as illustrated in lecture 17 regarding concerns over the misuse of advances in neuroscience. The section ends with a lecture that reviews the various papers that have recently discussed regulation of the security implications of the life sciences.

The final two sections of the EMR continue this theme of national and international regulation and are set out as follows:

Section $D$ of the EMR

19. International regulation of biotechnology

32 Revill, J. 2009, Biosecurity and bioethics education: A case study of the UK context, Wellcome trust Dual-Use Bioethics Group, University of Bradford, available: www.dual-usebioetics.net. 
20. National implementing legislation

Section E of the EMR

21. The web of prevention

Looking back at the list of specific suggestions agreed by State Parties to the BTWC in 2008, we would argue that we have covered most. Lectures cover the risks of misuse, the moral and ethical obligations of life scientists, give guidance on the types of activities which could be contrary to the aims of the Convention, and provide accessible teaching materials. Therefore, what else needs to be done?

\section{Increasing Efficiency through Networks}

One way to build on the work described here is to carry out more surveys of education provision in the university sector in different countries. These surveys, particularly if carefully followed up by questionnaire, telephone and email, inevitably provide a list of life-science lecturers who are interested in bringing issues of biosecurity and the dual-use dilemma into their courses. By assisting the development of country and regional networks on the basis of these contacts it should be possible to generate a much faster development and uptake of material suitable for different countries and regions. Such an approach would also fit with the States Parties agreement on the value of education efforts being integrated into existing international, regional and national activities.

As shown in a recent report from the US National Academies on 'Ethics Education and Scientific and Engineering Research: What's Been Learned? What Should Be Done?' dual-use bioethics developments will fit within a broader effort to develop ethics education. ${ }^{33}$

What is also clear is that these wider developments, whilst showing some advances in understanding how to proceed best in engaging students, have not yet found an adequate means of evaluating the impact of teaching on later ethical behaviour. The report points out that:

Attempts to evaluate and improve ethics education for scientific and engineering research and practice are just beginning. However, they do show that even though immediate results of some programmes are

33 National Academies 2009, Ethics Education and Scientific and Engineering Research: What's Been Learned? What Should Be Done? Summary of a workshop, Washington, DC: National Academies Press. 
positive, circumstances and pressures can overwhelm graduate students, postdoctoral fellows, and junior-faculty and researchers and undermine results.

In the longer term, attention to evaluation of the impact of dual-use bioethics education will be central to supporting the prohibition embodied in the BTWC.

More immediately, a further chance of improving efficiency will arise at the 2011 Seventh Review Conference of the BTWC because just after the specific suggestions on education (and codes of conduct) discussed above, paragraph 31 suggested that: 'State Parties are encouraged to inform the Seventh Review Conference of, inter alia, any actions, measures or other steps they may have taken on the basis of the discussions...in order to facilitate...decisions on further action.'

Therefore, if networks of life scientists concerned with implementing dualuse bioethics education can be established in different countries and regions, and if they carefully evaluate their efforts, the results could be applied rapidly elsewhere to help quickly close the education gap.

\section{What Should Be Done Now?}

Even if all of what has been discussed in this chapter were achieved it would still leave a great deal needing to be done. One specific point in the 2008 agreement amongst States Parties to the BTWC seems particularly important to us: trainthe-trainer programmes, being an important capacity-building initiative in developing a worldwide culture of responsibility amongst life scientists. In regard to this objective, we believe rapid progress can be made through the use of modern technology.

\section{Train-the-Trainer}

In order to facilitate efficient and effective engagement across a range of lifescience constituencies worldwide we developed an expert-level online distancelearning train-the-trainer programme in dual-use bioethics (biosecurity) education. The original iteration of this module consisted of two key elements: 1. the EMR described above, together with 20 expert-level scenarios that introduce users to examples of the complex bioethical dilemmas that have confronted life-science research; and 2. a range of innovative electronic online distance-learning technologies that facilitate outreach on a worldwide basis.

The aim of the module was to introduce educators to the concepts in bioethics and biosecurity education by developing awareness and understanding of a 
range of dual-use ethical issues arising from the impact of science and technology on society. The module provided the opportunity to develop knowledge of approaches that give a defence for ethical decisions or recommendations regarding dual-use technologies. Educators were guided through the lecture series by a trainer. Participants were introduced to scenarios where the results of well-intentioned scientific research can be used for both good and harmful purposes which have given, or may in future give, rise to what is now widely known as the 'dual-use dilemma', providing the opportunity to analyse in depth the ethical dilemmas these scenarios raise. Central to this is the importance and role of ethics in informing the debate. The programme was intended to have an applied, practical dimension in that its aim was to enable and facilitate more bioethical research into dual-use issues, and help develop policies and practices that might prevent the misuse of knowledge generated through biomedical research.

The methodological approach relating to the delivery and implementation of this module was developed with UK academic standards in mind, so that the module would furnish participants with knowledge and understanding to review and appraise ethical theories and methods relevant to dual-use bioethics and recognise and discuss ways in which the application of ethics methodologies resolves or leaves unresolved questions relating to dual-use issues. In relation to subject-specific skills, the module would facilitate educators' organisation and synthesis of ideas and questions relevant to assessing ethical dilemmas in specific dual-use issues affecting humans, animals and plants generally, and across a select range of life-science sub-disciplines of relevance including human biology, zoonotic diseases, phytopthology, biotechnology, DNA synthesis, drug control, genomics, genetic engineering and genetic modification, immunology, nanotechnology, neuroscience, scientific freedom, synthetic biology, whistleblowing, and processes relating to ethical review. As to personal transferable skills, educators would be able to evaluate and integrate data from a variety of sources and express ideas clearly, both verbally and in writing; and communicate effectively in an online environment using a range of media.

\section{Technologies}

The module was designed to facilitate participation in lectures, seminars, and discussion groups that would all take place online. A novel approach to online distance learning was adopted. This utilised online distance-learning technologies that facilitate the delivery, viewing of, and participation in lectures by real-time video transmission. With this approach participants can see a live video transmission of the teacher, and the teacher can guide the participants through respective online sessions with the support of a range of online teaching technologies and visual aids. Together with the lecture, these 
can be broadcast simultaneously, including PowerPoint presentations, wordprocessing files, graphical images, as well as audio and video. Participants with video cameras can be invited by the teacher to join live online 'face-to-face' discussion and the latter can be viewed online by all members. Those with the capability to transmit audio can raise a (virtual) hand, be invited by the teacher to join live online discussion, and can communicate this way with all of those taking part, regardless of geographical location. Participants without video and audio capability can follow the class online and communicate with the teacher by typing questions via their keyboard. The classes can be recorded and viewed online subsequently.

Working in a fully supported online-learning community, members are able to communicate and interact with peers, developing their practice through sustained reflection and involvement in a range of activities and scenarios. Participants are encouraged to bring their own ideas and experiences to the course, sharing these with peers to contextualise their knowledge and understanding in ways that will help them, as life-science professionals, to meet the ethical challenges thrown up by dual use. As well as participation in a vibrant academic (socialnetwork) web-group where interaction on coursework-related topics between tutors, moderators and students takes places, members undertake independent reading and research. Participants benefit from a supportive and interactive online web-based learning community and work both independently to produce a coursework assignment, as well as in online groups to produce a significant group-work course assignment.

\section{Conclusion: Recommendations for the BTWC}

As has been made clear, a major effort will be required to raise awareness levels amongst life scientists and develop a culture of responsibility around the dualuse implications of research. Whilst concerns raised by high-level reviews of scientific oversight of dual-use research are becoming assimilated into the terms and conditions associated with the funding of life-science research, this is a long-term initiative and will necessarily involve a broad range of constituencies. As set out by Mathews and Webb, ${ }^{34}$ two practical suggestions would assist in sustaining interest in this area. The first is that States Parties could report to the Seventh Review Conference of the BTWC in 2011 on progress on implementation and capacity building in dual-use/biosecurity education. The second is that

34 Mathews, R. J. and Webb, J. M. 2009, 'Awareness-raising, Education and Codes of Conduct within the Framework of the BWC', Chapter 9, BWPP Biological Weapons Reader, McLaughlin, K. and Nixdorff, K. (eds). 


\section{Education and Ethics in the Life Sciences}

the confidence-building mechanism (CBM) could be extended by appending progress reports on implementation and capacity building in education for life scientists to annual CBM reports. 